\title{
The study of TrkB pathway on vascular endothelial permeability by regulating the synthesis of $\mathrm{VE}$-cadherin and inhibiting the degradation of VE-cadherin.
}

\author{
Weichao Shan ${ }^{1 \#}$, Chunling Li ${ }^{2 \#}$, Chao Liu ${ }^{1}$, Fei Shi ${ }^{1}$, Ruijuan Wang ${ }^{1}$, Lixian Sun ${ }^{1}$, Zhenjiang Ding ${ }^{*}$ \\ ${ }^{1}$ Department of Cardiology, Affiliated Hospital of Chengde Medical University, Chengde City, Hebei Province, PR China \\ ${ }^{2}$ General Internal Medicine, Wuwei City People's Hospital of Gansu Province, Wuwei City, Gansu Province, PR China \\ \#These authors contribute equally to this work
}

\begin{abstract}
Objective: To investigate the effects and mechanism of tyrosine kinase receptor B (TrkB) on the vascular endothelial permeability.

Methods: The counts of FITC-labeled dextran and T cells which were entered through the endothelial monolayer into the lower chamber were detected by Transwell chamber. The TrkB gene and VEcadherin gene were cloned into a vector, which was transfected into 293T cells. The expressions of VEcadherin mRNA and Est1 were detected by real-time PCR, and the expressions of VE-cadherin protein were detected by Western blot.

Results: TrkB knockdown could significantly increase the FITC-labeled dextran diffusion, VE-cadherin gap formation and migration of $T$ cells across the endothelial monolayer. Meanwhile, TrkB knockdown also decreased the expressions of VE-cadherin mRNA and protein. Importantly, he FITC-labeled dextran diffusion, VE-cadherin gap formation and migration of $T$ cells regulated by TrkB knockdown were all prevented by overexpressing VE-cadherin. Besides, TrkB knockdown also decreased the expression of Est1. The results above were different of the control group, $P<0.05$.

Conclusion: There are significant evidences that TrkB could protect the vascular endothelial permeability by regulating the synthesis of VE-cadherin and inhibiting the degradation of VE-cadherin.
\end{abstract}

Keywords: Tyrosine kinase receptor B, VE-cadherin, Vascular endothelial permeability, Endothelial barrier, TrkB pathway.

Accepted on November 29, 2017

\section{Introduction}

Tyrosine kinase receptor B (TrkB) is one of the high affinity receptors of brain derived neurotrophic factors. It is mostly expressed in the cardiovascular and nervous system, and closely related to endothelial cell adhesion $[1,2]$. When both TrkB and E-cadherin are expressed, endothelial cells may be aggregated into clusters, and then involved in the protection of the vascular endothelial barrier [3,4]. If vascular endothelial permeability is abnormal, it may cause lipid components, monocytes or smooth muscle cells in the plasma to migrate to the intima, thereby damaging the blood vessels or leading to atherosclerosis [5].

This study investigated the mechanism of action on TrkB pathway participating in the protection of vascular endothelial permeability by using $A p o E$ gene to knockout mouse models and human vascular endothelial cells. It is hereby reported as follows.

\section{Materials and Methods}

\section{Main instruments and reagents}

Ethical approval was given by the medical ethics committee of Affiliated hospital of Chengde medical college with the following reference number: 2016016.GTR16-2 type high speed tabletop refrigerated centrifuge(purchased from Beijing Time Beili centrifuge Co., Ltd.); real-time quantitative PCR instrument (purchased from MJ Research company in USA); gel imager (purchased from Furi technology Co., Ltd.); UV-8000 ultraviolet spectrophotometer (purchased from Shanghai precision instrument and meter company); multifunctional enzyme indicator (purchased from Bio-Rad company in Japan); CX41 inverted optical microscope and OLS4100 laser scanning confocal microscope(purchased from OLYMPUS medical company in Japan).

TrkB genotype detection probe(purchased from Shanghai Jikang company); PCR primers were synthesized and provided 
by Shanghai Shenggong bio Engineering Corporation ltd.; $\beta$ actin monoclonal antibodies, Tubulin monoclonal antibodies, TrkB monoclonal antibodies, endothelial cadherin antibodies (C19 and BV9), Etsl antibodies, etc. (purchased from Abcam company in the United States); BCA protein concentration assay Kit (purchased from Boshide Bioengineering Co., Ltd. in China); RNA extraction, reverse transcription, PCR reagents and gene cloning reagents, etc. (purchased from OMEGA biological company in USA); small interfering RNA was synthesized and supplied by Shanghai Shenggong bio engineering Co., Ltd.; the DMEM medium was purchased from GIBCO Corporation in the United states; Trypsin-EDTA cell digestive juice $(0.25 \%)$ was purchased from Beijing Niuyin Huaxin Technology Development Co., Ltd.; (FBS) Fetal Bovine Serum was purchased from ThermoFisher company in the United States; Human aortic endothelial cells(purchased from Shanghai Zhongqiao Xinzhou Biotechnology Co., Ltd.).

\section{Animal subject}

Apo-/-E gene was used to knockout mice with clean grade, 18-20 g, and provided by Beijing Weitong Lihua experimental animal center, fed in SPF grade animal room in this unit with room temperature $\left(24 \pm 5^{\circ} \mathrm{C}\right)$, lighting on alternating day and night, and animal subjects were free for water and food intake.

\section{Experimental method}

Cell culture: Human aortic endothelial cells were cultured in endothelial cell medium, besides, Hela cells and 293T cells were cultured in DMEM medium which was added by fetal bovine serum. The cells were cultured in $5 \% \quad \mathrm{CO}_{2}$ cell incubator at $37^{\circ} \mathrm{C}$. Cell medium was replaced after $24 \mathrm{~h}-48 \mathrm{~h}$, and conducted passage once in $48 \mathrm{~h}$.

Analysis of in vitro paracellular permeability: Human aortic endothelial monolayer cells in single layer were inoculated into a Transwell chamber enclosed by collagen in 4 well plates and added by FITC labeled dextran $(1 \mathrm{mg} / \mathrm{ml}) .50 \mu \mathrm{l}$ medium in lower chamber was sampled every $30 \mathrm{~min}$ and $50 \mu \mathrm{l}$ fresh culture medium was added at the same time. The fluorescence value was detected by enzyme analyzer.

Transendothelium migration detection: Human aortic endothelial monolayer cells in single layer were inoculated into a Transwell chamber enclosed by collagen in 6 well plates, $1 \mathrm{ml}$ $\mathrm{T}$ cells stimulated by $6 \times 10^{5} / \mathrm{ml}$ phytohemagglutinin were added into the upper chamber, and the number of $\mathrm{T}$ cells migrating to the lower chamber after $24 \mathrm{~h}$ was counted, and then the migration rate was calculated.

RNA interference: TrkB small interfering RNA sequence: 5'AACCACUGGGAUCAGCUGGUGUU-3' and 'AACACCAGCUGAUCCCAGUGGUU-3' 5. Negative control of small interfering RNA sequence: 5'UUCUCCGAACGUGUCACGU-3' and 'ACGUGACACUUCGGAGAA-3 5'.
Construction of lentiviral vector: TrkB, cDNA and cadherin cDNA were respectively cloned into lentiviral vector such as pccl.PGK.Tetlinker.WPRE, and then stably transfected into 293 T cells.

Real-time quantitative PCR: the $20 \mu \mathrm{l}$ reaction system was placed in thermostatic water bath at $37^{\circ} \mathrm{C}$ for $60 \mathrm{~min}, 85^{\circ} \mathrm{C}$ for $5 \mathrm{~s}$. Add deionized water to $100 \mu \mathrm{l}$, and take $2 \mu \mathrm{l}$ for PCR from various reaction holes. $20 \mu \mathrm{l}$ PCR reaction system was prepared in ice bath, pre denature at $95^{\circ} \mathrm{C}$ for $30 \mathrm{~s}, 60^{\circ} \mathrm{C}$ for 30 $\mathrm{s}$, and recycle for 45 times. The primer sequences are as follows: TrkB (upstream primer: 5'TGGCATCACCAACAGTCAGC-3'; downstream primer: 5'CCTCCACGCAGACGCCATA-3'); human endothelial cadherin (upstream primer: 5'AGCCCAAAGTGTGTGAGAACG-3'; downstream primer: 5'-GAGATGACCACGGGTAGGAAG-3'); mouse endothelial cadherin: (upstream primer: 5'AGCAGGGAAACATCTATAACG-3'; downstream primer: 5'CTTGAACTTTGGGTTTACTGG -3').

Western blot: The preparation of protein samples; gel electrophoresis of protein samples; trarsmembrane; close; adding primary antibodies for incubation; add secondary anti for incubation; developing.

\section{Statistical processing}

These data were processed by using SPSS19.0 statistical software; the data were expressed by the average and standard deviation; t-test or Mann-Whitney $U$ test were used for variables between various groups; continuous variables among multiple groups were analysed by ANOVA analysis, categorical variables were tested by using chi square test, and $\mathrm{P}<0.05$ indicated the difference was statistically significant.

\section{Result}

\section{The effect of TrkB knockout on dextran through endothelial cells}

TrkB knockout obviously increased the degree of crossendothelial diffusion of FITC labeled dextran. TrkB iRNA interference and TrkB+LV-VEcad and iRNA interference were added into a Transwell chamber lined with a single layer of human aortic endothelial cells, and the permeability of endothelial cells was significantly higher than that of the negative control group, with statistical difference, $\mathrm{P}<0.05$. However, after adding TrkB+LV-VEcad iRNA interference, the permeability of endothelial cells was obviously larger than that of only adding TrkB and iRNA, and the permeability was statistically different, $\mathrm{P}<0.05$, Figure 1 .

\section{Effect of TrkB knockout on migration of T cells across endothelial cells}

TrkB knockout significantly increased the ability of $\mathrm{T}$ cells migrating across the monolayer endothelial, whereas the ability of $\mathrm{T}$ cells migrating across the endothelial was closely 
The study of TrkB pathway on vascular endothelial permeability by regulating the synthesis of VE-cadherin and inhibiting the degradation of VE-cadherin

associated with inflammatory responses. TrkB iRNA interference, TrkB+LV-Con iRNA interference, TrkB+LVVEcad iRNA interference were respectively added into a Transwell chamber lined with a single layer of human aortic endothelial cells, and the ability of $\mathrm{T}$ cells migrating in TrkB iRNA interference and TrkB+LV-Con iRNA interference groups was significantly higher than that of the control group, with statistical difference, $\mathrm{P}<0.05$. However, after adding TrkB + LV-VEcad iRNA interference, at the same time, higher than that of TrkB+LV-VEcad iRNA interference group with statistical difference, $\mathrm{P}<0.05$, Figure 2 .

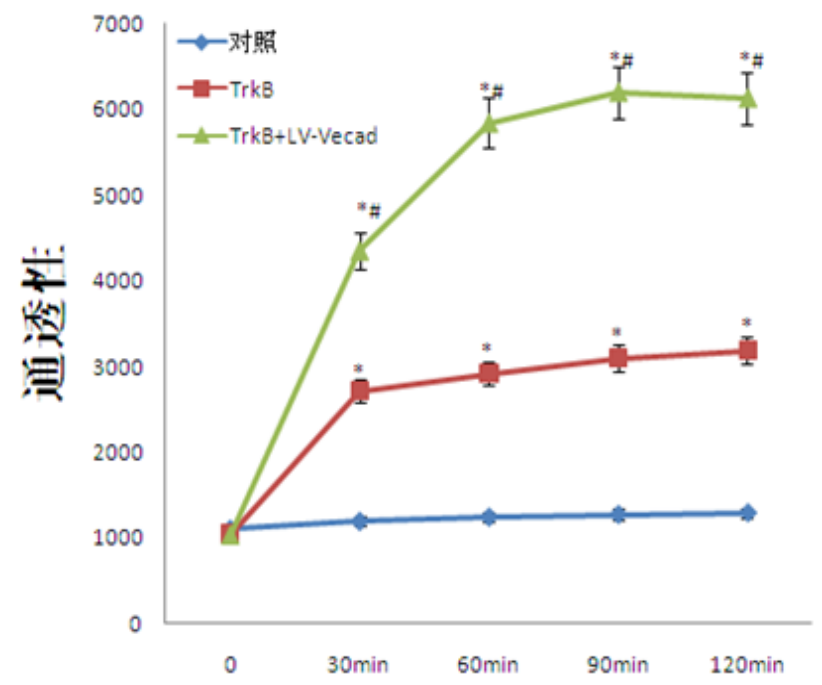

Figure 1. The effect of TrkB knockout on FITC labeled dextran through endothelial cells and the mediation function of cadherin in vitro detection ("compared with control group, $P<0.05$; " compared with TrkB iRNA interference group, $P<0.05$ ).

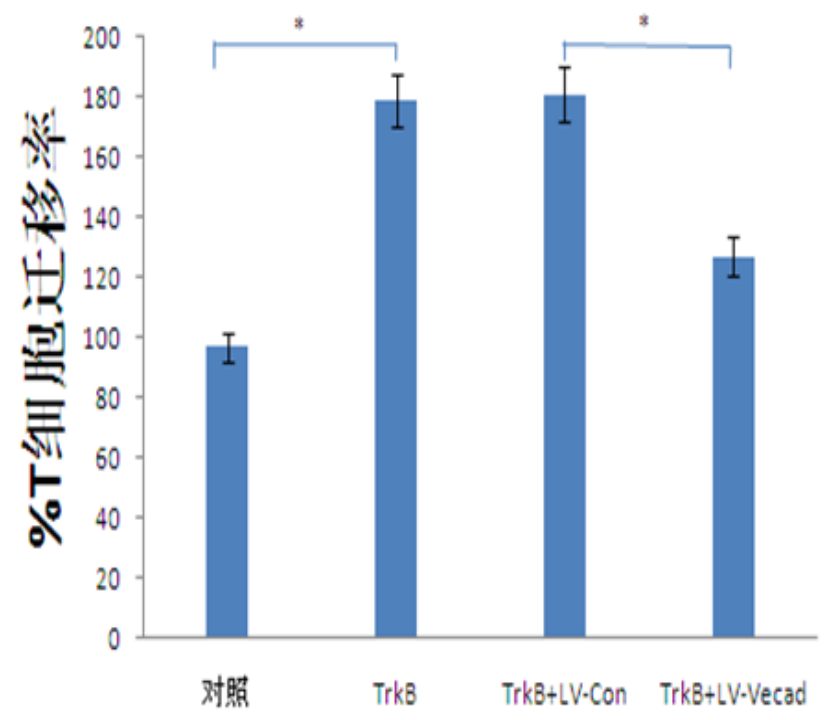

Figure 2. The effect of TrkB knockout on T cells migrating across endothelial cells and the mediation function of cadherin in vitro detection.

\section{Effects of TrkB knockout on the formation of cadherin gap in endothelial cells}

TrkB knockout could obviously promote the formation of cadherin gap in endothelial cells, and the formation of cadherin gap was closely related to the migration ability of $\mathrm{T}$ cells across endothelial cells. TrkB iRNA interference and TrkB + LV-VEcad iRNA interference were respectively added into a Transwell chamber lined with a single layer of human aortic endothelial cells, and the formation rate of cadherin gap in endothelial cells in TrkB iRNA interference group was significantly higher than that of the control group and TrkB + LV-VEcad iRNA interference group with statistical difference, $\mathrm{P}<0.05$, Figure 3 .

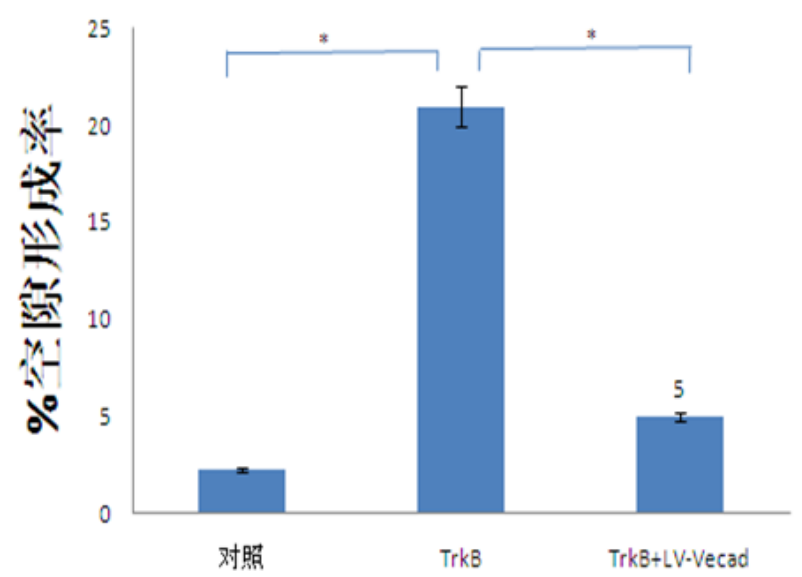

Figure 3. The effect of TrkB knockout on the formation of cadherin gap in endothelial cells in vitro detection.

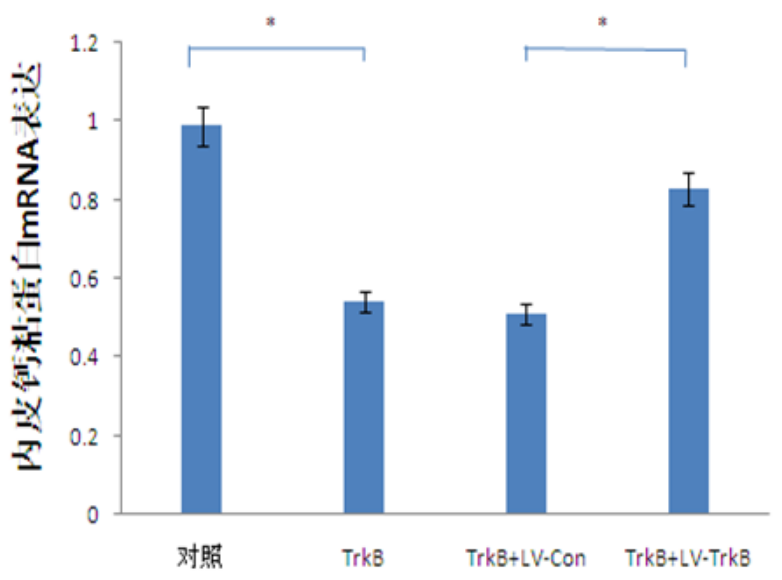

Figure 4. The effect of TrkB knockout on the expression level of cadherin $m R N A$ in endothelial cells in vitro detection.

\section{The effect of TrkB knockout on the expression level of cadherin mRNA in endothelial cells}

TrkB knockout obviously inhibited the expression of cadherin mRNA in endothelial cells. TrkB iRNA interference, TrkB + LV-Con iRNA interference and TrkB+LV-TrkB iRNA interference were respectively added into a Transwell chamber 
lined with a single layer of human aortic endothelial cells, and the expression amount of cadherin mRNA in endothelial cells in TrkB iRNA interference group and TrkB+LV-Con iRNA interference group were significantly lower than that of the control group as well as TrkB+LV-TrkB iRNA interference group with statistical difference, $\mathrm{P}<0.05$ as seen in Figure 3. It indicated that the overexpression of exogenous TrkB could inhibit the decrease of cadherin mRNA expression in endothelial cells, as shown in Figure 4.

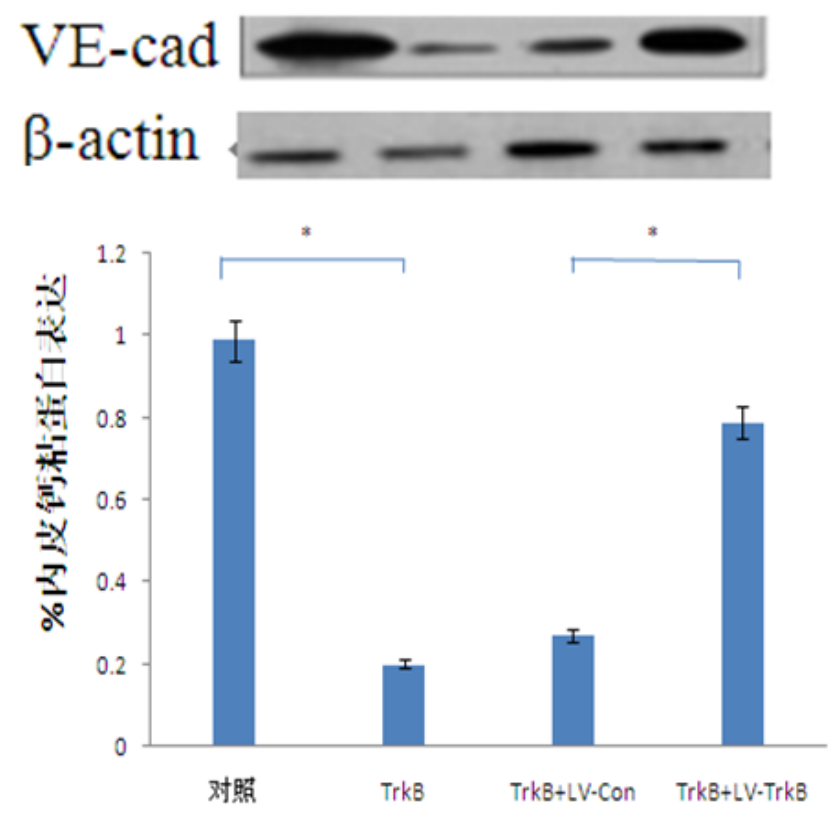

Figure 5. The effect of TrkB knockout on the expression level of cadherin in endothelial cells in vitro detection.

\section{The effect of TrkB knockout on the expression level of cadherin in endothelial cells}

TrkB knockout obviously inhibited the expression of cadherin in endothelial cells. TrkB iRNA interference, TrkB+LV-Con, iRNA interference and TrkB+LV-TrkB iRNA interference were respectively added into a Transwell chamber lined with a single layer of human aortic endothelial cells, and the expression amount of cadherin in endothelial cells in $\operatorname{TrkB}$ iRNA interference group and TrkB+LV-Con iRNA interference group were significantly lower than that of the control group as well as TrkB + LV-TrkB iRNA interference group with statistical difference, $\mathrm{P}<0.05$, the which was consistent with the vitro detection results about the effect of TrkB knockout on the expression of cadherin mRNA in endothelial cells. However, overexpression of exogenous TrkB could prevent the decrease in cadherin expression, as shown in Figure 5.

\section{The effect of TrkB knockout on the proliferation of endothelial cells}

Because the TrkB pathway could promote the proliferation of endothelial cells, therefore, we further detected the effect of TrkB knockout and overexpression on the proliferation of endothelial cells. The results showed that both the knockout and overexpression of TrkB had no effect on the proliferation of endothelial cells, $\mathrm{P}>0.05$, as seen in Figure 6 .

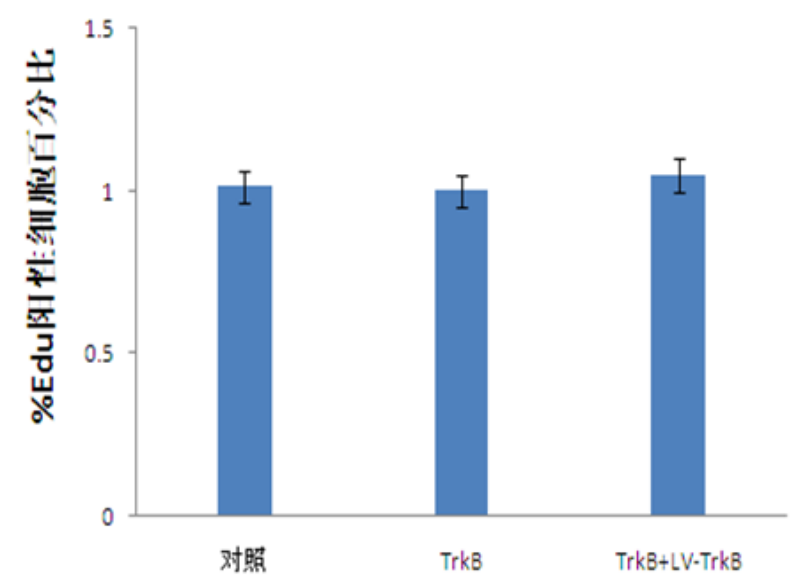

Figure 6. The effect of TrkB knockout on proliferation of endothelial cells in vitro detection.

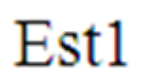

$\beta$-actin

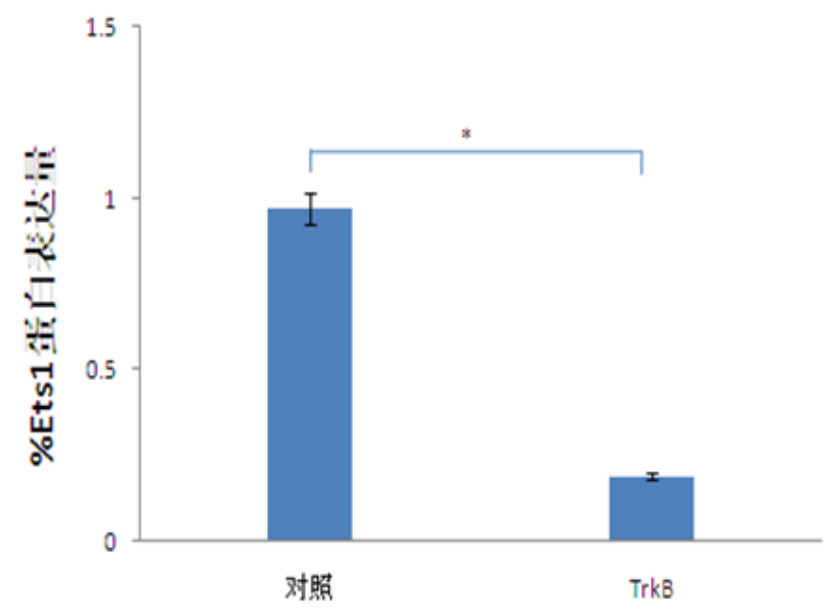

Figure 7. The effect of TrkB knockout on the expression level of endothelial Est1 in vitro detection.

\section{The effect of TrkB knockout on the expression of endothelial Est1}

For Est is closely related to the expression of cadherin, while Est1 is a Est transcription factor which has the largest expression amount in endothelial cells. Therefore, through detecting the effect of TrkB knockout on the expression of Est1, it indirectly showed the relationship between TrkB and cadherin. The results confirmed that TrkB knockout obviously inhibited the expression amount of Estlwith significant differences, $\mathrm{P}<0.05$, Figure 7 . 
The study of TrkB pathway on vascular endothelial permeability by regulating the synthesis of VE-cadherin and inhibiting the degradation of VE-cadherin

\section{Discussion}

The basic function of vascular endothelium is a selective barrier to cells, body fluids and proteins, etc. However, the blood flow of capillaries varies when stimulated by diseases, trauma and other pathological changes, endothelial cells are attacked by inflammatory cytokines, causing injury or apoptosis. And the tight junction proteins are destroyed, thereby increasing the permeability of the vascular endothelium [6,7]. Abnormal permeability of blood vessel endothelium not only leads to capillary microleakage, but also participates in the occurrence and development of atherosclerosis, and even causes plaque rupture [8,9].

The channels of vascular endothelial permeability include cells collateral pathway mediated by the adhesion and connection between endothelial cells and intracellular pathway mediated by vesicae [10]. Besides, the lipoprotein and other biological macromolecules mainly cross the vascular endothelial layer through cell collateral pathway, therefore, the adhesion ability of endothelial cell determines vascular permeability $[11,12]$. TrkB can be highly integrated with brain-derived neurotrophic factors and participates in various physiological processes of neurons in the central and peripheral nervous system [2]. Meanwhile, TrkB is also highly expressed in the cardiovascular system and is closely related to adhesion function of endothelial cell [3]. Thus, this study aims to investigate the impact of TrkB knockout on endothelial cadherin. The results confirm that TrkB is closely related to the expression of vascular endothelial cadherin, and promotes the synthesis of cadherin by inducing and activating the expression of Est1 transcription factors. Knockout of TrkB significantly increases the transendothelium diffusion degree of FITC labeled dextran, the ability of $\mathrm{T}$ cells' transendothelial monolayer migration and the formation of cadherin gap in endothelial cells as well as promoted endothelial cell proliferation and at the same time, significantly inhibits the expression of vascular endothelial cadherin. This suggests that adhesion and connection mediated by endothelial cadherin is critical for the protection of vascular endothelium permeability. Studies have shown that the degradation of endothelial cadherin and the increase of vascular endothelial permeability are closely related to the formation of atherosclerosis [13,14]. However, studies have also shown that after intercellular connections mediated by endothelial cadherin are destructed, cadherin can repair it rapidly, thus re-establish the connections $[15,16]$. The increase of vascular permeability is not only related to the degradation of cadherin, but also to the synthesis ability of cadherin $[17,18]$. The results of this study confirm that TrkB not only inhibits the cleavage of cadherin, but also regulates the synthesis of cadherin as well as protects the permeability of vascular endothelium from damage.

In summary, this study confirms that TrkB/Est1/endothelial cadherin pathway is involved in the mediating of vascular endothelial permeability. TrkB promotes the synthesis of cadherin, inhibits the degradation of cadherin as well as protects the permeability of vascular endothelial through inducing and activating the expression of Est1 transcription factors.

\section{References}

1. Burk K, Murdoch JD, Freytag S. EndophilinAs regulate endosomal sorting of BDNF- TrkB to mediate survival signaling in hippocampal neurons. Sci Rep 2017; 7: 2149.

2. Yuan JH, Pan F, Chen J. Neuroprotection by plumbagin involves BDNF-TrkB-PI3K/Akt and ERK1/2/JNK pathways in isoflurane-induced neonatal rats. J Pharm Pharmacol 2017; 69: 896-906.

3. Davila LF, Dias VT, Vey LT, Milanesi LH, Roversi K, Emanuelli T, Burger ME, Trevizol F, Maurer HL. Toxicological aspects of interesterified fat: Brain damages in rats. Toxicol Lett 2017; 276: 122-128.

4. Fang MS, Li X, Qian H, Zeng K, Ye M, Zhou YJ, Li H, Wang XC, Li Y. Ï-3PUFAs prevent MK-801-induced cognitive impairment in schizophrenic rats via the CREB/ BDNF/TrkB pathway. J Huazhong Univ Sci Technolog Med Sci 2017; 37: 491-495.

5. Shan C, Wei J, Hou R. Schwann cells promote EMT and the Schwann-like differentiation of salivary adenoid cystic carcinoma cells via the BDNF/TrkB axis. Oncol Rep 2016; 35: 427-435.

6. Gotz R, Sendtner M. Cooperation of tyrosine kinase receptor TrkB and epidermal growth factor receptor signaling enhances migration and dispersal of lung tumor cells. PLoS One 2014; 9: 100944.

7. Ricci A, De Vitis C, Noto A. TrkB is responsible for EMT transition in malignant pleural effusions derived cultures from adenocarcinoma of the lung. Cell Cycle 2013; 12: 1696-1703.

8. Jiang $\mathrm{H}$, Huang $\mathrm{S}$, Li X. Tyrosine kinase receptor $\mathrm{B}$ protects against coronary artery disease and promotes adult vasculature integrity by regulating Ets1-mediated VEcadherin expression. Arterioscler Thromb Vasc Biol 2015; 35: 580-588.

9. Jiang $\mathrm{H}$, Huang $\mathrm{S}$, Li $\mathrm{X}$. Endothelial tyrosine kinase receptor $\mathrm{B}$ prevents $\mathrm{VE}$-cadherin cleavage and protects against atherosclerotic lesion development in ApoE-/- mice. Oncotarget 2015; 6: 30640-30649.

10. Bailey JJ, Schirrmacher R, Farrell K. Tropomyosin receptor kinase inhibitors: an updated patent review for 2010-2016Part II. Expert Opin Ther Pat 2017; 1-19.

11. Wurzelmann M, Romeika J, Sun D. Therapeutic potential of brain-derived neurotrophic factor (BDNF) and small molecular mimics of BDNF for traumatic brain injury. Neural Regen Res 2017; 12: 7-12.

12. Li X, Chen C, Yang X, Wang J. Acupuncture improved neurological recovery after traumatic brain injury by activating BDNF/TrkB pathway. Evid Based Complement Alternat Med 2017; 2017: 8460145.

13. Matsuda S, Fujita T, Kajiya M. Brain-derived neurotrophic factor prevents the endothelial barrier dysfunction induced 
by interleukin- $1 \beta$ and tumor necrosis factor-a. J Periodontal Res 2015; 50: 444-451.

14. Katsuhiro T, Yusuke O, Akihiro K. Brain-derived neurotrophic factor inhibits intercellular adhesion molecule-1 expression in interleukin-1 $\beta$-treated endothelial cells. Cell Biochem Biophys 2016; 74: 399-406.

15. Zhang Z, Zhang Y, Zhou Z. BDNF regulates the expression and secretion of VEGF from osteoblasts via the TrkB/ ERK1/2 signaling pathway during fracture healing. Mol Med Rep 2017; 15: 1362-1367.

16. Tao C, Yu W, Wang Y. Brain-derived neurotrophic factor increases synaptic protein levels via the MAPK/Erk signaling pathway and Nrf2/Trx axis following the transplantation of neural stem cells in a rat model of traumatic brain injury. Neurochem Res 2017; 1-11.

17. Pandya CD, Hoda N, Crider A. Transglutaminase 2 overexpression induces depressive-like behavior and impaired TrkB signaling in mice. Mol Psychiatry 2017; 22: 745-753.

18. Boulle F, Velthuis H, Koedam K. Behavioral and neurochemical characterization of TrkB-dependent mechanisms of agomelatine in glucocorticoid receptorimpaired mice. Eur Neuropsychopharmacol 2016; 26: 65-77.

\section{*Correspondence to}

Zhenjiang Ding

Department of Cardiology

Affiliated Hospital of Chengde Medical University

PR China 